\title{
Síndrome cardiopulmonar por Hantavirus: utilidad de la monitorización con el sistema PiCCO
}

\author{
Carlos Romero $\mathbf{P}^{1}$, Max Andresen $\mathbf{H}^{1}$, O rlando Díaz $\mathbf{P}^{1}$, \\ Vinko Tomicic $F^{1}$, Fernando Baraona $R^{2}$, Marcelo Mercado $F^{1}$, \\ Carlos Pérez $C^{2}$, Patricio Downey $C^{3}$, Alberto Dougnac $L^{1}$. \\ PiCCO (Pulse contour cardiac \\ output) system for monitoring \\ Hantavirus Cardiopulmonary \\ Syndrome: report of one case
}

We report a 68 years old man, farmer from the metropolitan region, admitted with a Hantavirus Cardiopulmonary Syndrome. The diagnosis was made using serologic test and was later confirmed by the Public Health Institute. He evolved to an early multiple organ failure, requiring high concentrations of oxygen and invasive ventilatory assistance, vasopressor drugs and renal replacement therapy. Swan Ganz and PiCCO were used simultaneously for hemodynamic monitoring. Treatment consisted in global support therapy, antimicrobial therapy and systemic corticosteroids. Intrathoracic blood volume was a more reliable parameter than pulmonary capillary wedge pressure for the assessment of preload. As expected in situations of increased vascular permeability, there was an increase in extravascular lung water. There was a good correlation between extravascular lung water and oxygenation parameters $\left(\mathrm{PaO}_{2} / \mathrm{FiO}_{2}\right.$ and oxygenation index). PiCCO system may become a helpful tool in the management of patients with Hantavirus Cardiopulmonary Syndrome (Rev Méd Chile 2003; 131: 1173-78)

(Key Words: Cardiac output; Hantavirus pulmonary syndrome; Multiple organ failure)

Recibido el 9 de mayo, 2003. Aceptado en versión corregida el 17 de julio, 2003.

${ }^{1}$ Programa de Medicina Intensiva.

${ }^{2}$ Departamentos de Medicina Interna y ${ }^{3}$ Nefrología. Facultad de Medicina. Hospital Clínico de la Pontificia Universidad Católica de Chile. Santiago de Chile

$\mathrm{E}$ l virus Hanta se reconoció por primera vez en 1993 como el agente causal de una severa enfermedad en seres humanos en el continente americano $^{1}$.

Correspondencia a: Dr. Carlos Romero P. Marcoleta 367, Santiago, Casilla 114-D. E-mail: cmromero@puc.cl
Aunque inicialmente se resaltó el compromiso pulmonar, investigadores de la Universidad de Nuevo México describieron una importante alteración cardíaca en los casos más graves ${ }^{2-4}$.

La disfunción cardiopulmonar es variable y va desde hipoxemia leve con hemodinamia estable 
hasta falla respiratoria rápidamente progresiva y shock $^{5-8}$. En aquellos pacientes afectados por el síndrome cardiopulmonar por Hantavirus (SCPH), al igual que en otros pacientes críticos, el objetivo primario del tratamiento consiste en restaurar y mantener la perfusión tisular.

El sistema PiCCO ( output» Pulsion Medical Systems, Munich, Alemania), recientemente desarrollado e incorporado a la práctica clínica para el monitoreo hemodinámico de pacientes críticos, ha sido empleado en la valoración del débito cardíaco, agua pulmonar extravascular, volumen sanguíneo intratorácico y otros parámetros derivados, en pacientes cursando síndrome de distrés respiratorio agudo. Su empleo y utilidad en el monitoreo cardíaco y respiratorio del SCPH no ha sido documentado.
Comunicamos un caso de SCPH monitorizado con el sistema PiCCO y manejado de acuerdo a un algoritmo de soporte hemodinámico (Figura 1).

\section{CASO CLINICO}

Agricultor de 68 años, residente en la comuna de Peñaflor, con antecedentes de diabetes mellitus tipo 2 (DM 2) diagnosticada en 1994, en tratamiento irregular con hipoglicemiantes, e hipertensión arterial (HTA) diagnosticada en el año 2000, sin tratamiento.

Presentó un cuadro clínico de 7 días de evolución, caracterizado por mialgias, sensación febril, compromiso del estado general de intensidad progresiva, sin síntomas respiratorios asociados.

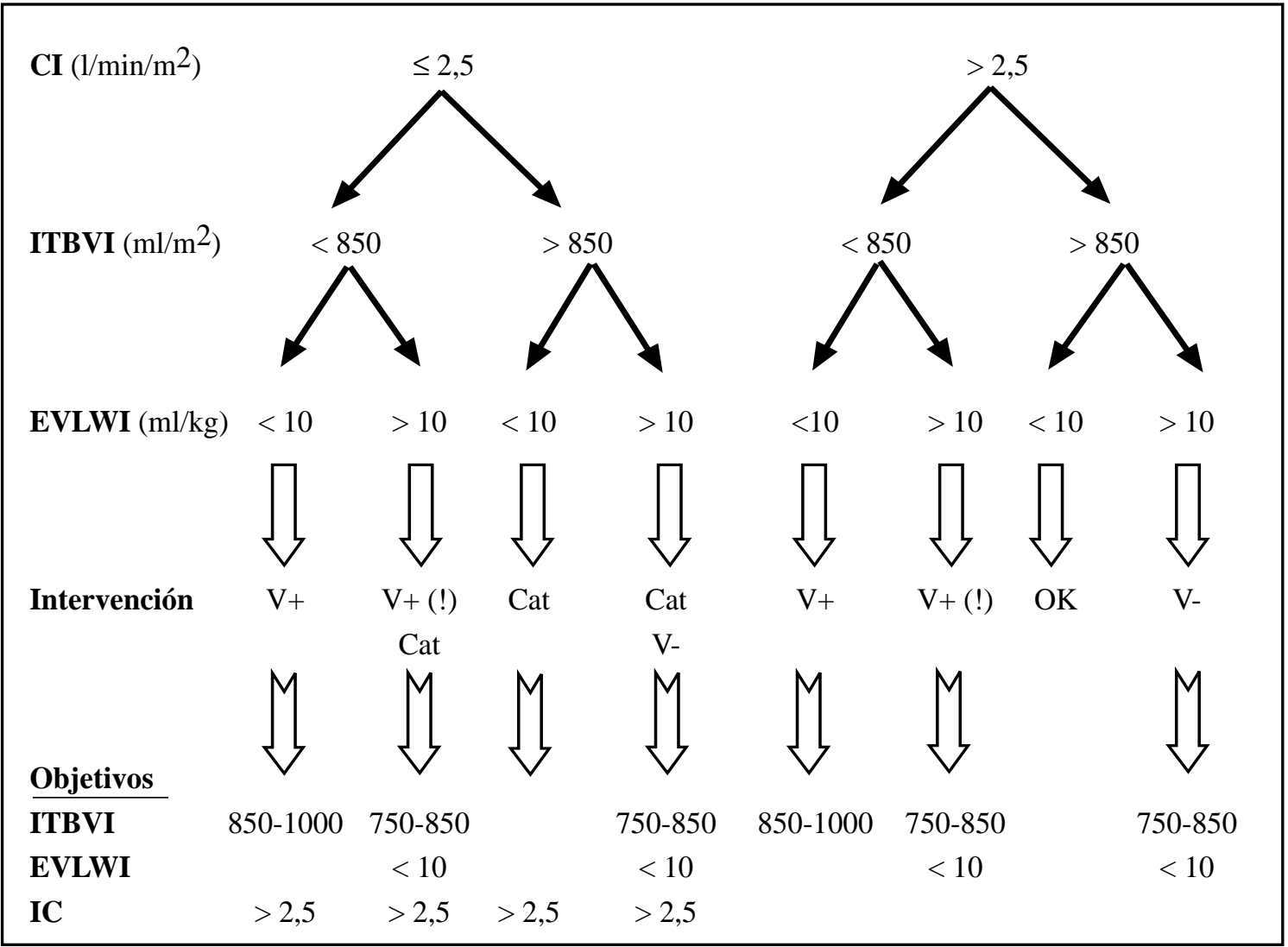

FIgURA 1. Algoritmo de manejo hemodinámico PiCCO*

V+: reposición de volumen, !: cuidadosamente, V-: depleción de volumen, Cat: catecolaminas.

*Modificado del algoritmo propuesto por el fabricante del sistema PiCCO. 
Cuarenta y ocho horas antes de su ingreso se agregaron deposiciones líquidas de alto volumen, sin elementos patológicos ni dolor abdominal. Fue llevado a un Servicio de Urgencia, donde se le encontró deshidratado, con insuficiencia respiratoria parcial, y compromiso cualitativo de conciencia, siendo derivado a nuestro centro. A su ingreso al Hospital Clínico de la Universidad Católica de Chile, se obtuvo el antecedente de haber estado días antes realizando la limpieza de una bodega que había permanecido cerrada. El paciente se encontraba hemodinámicamente estable, afebril, saturando 89-90\% con máscara facial al 30\%, oligoanúrico. Al examen destacaba: cianosis periférica, taquipnea $\left(30 \mathrm{x}^{\prime}\right)$, crépitos difusos, hepatoesplenomegalia. Laboratorio: hematocrito 50\%, leucocitos 9900 con presencia al frotis de inmunoblastos (Figura 2), plaquetas 96.000, VHS 23, PCR $35 \mathrm{mg} / \mathrm{dL}$, TP 77\%, TTPK 45", creatinina 2,78 mg/ dL, BUN $66 \mathrm{mg} / \mathrm{dL}$, pruebas hepáticas normales. Los gases arteriales mostraban una insuficiencia respiratoria parcial $\left(\mathrm{PaO}_{2} / \mathrm{FiO}_{2}: 56\right)$. En la radiografía de tórax se apreciaba infiltrado intersticial y alveolar, en zonas dependientes, con leve derrame pleural bilateral. Su cuadro respiratorio progresó rápidamente, por lo que se intubó y conectó a ventilación mecánica. Se ajustó PEEP hasta un nivel de $12 \mathrm{cmH}_{2} \mathrm{O}$ (mediante una curva presión/volumen por técnica de oclusión múltiple), consignándose una distensibilidad estática de $52 \mathrm{ml} / \mathrm{cmH}_{2} \mathrm{O}$. A las 3 horas de la intubación presentó deterioro hemodinámico, que requirió apoyo con dopamina y noradrenalina (dosis máximas $5 \mu \mathrm{g} / \mathrm{kg} / \mathrm{min}$ y 0,29 $\mu \mathrm{g} / \mathrm{kg} / \mathrm{min}$, respectivamente). En la radiografía de tórax se evidenció rápida progresión del infiltrado intersticial y alveolar bilateral. Se inició antibioticoterapia empírica de amplio espectro con cefotaximo y levofloxacino, y dada la alta sospecha de SCPH se agregó metilprednisolona $450 \mathrm{mg} /$ día. Evolucionó en forma tórpida con desarrollo de disfunción orgánica múltiple (hemodinámica, renal, respiratoria y hematológica (AAPACHE II 25 puntos y SOFA 12 puntos») dentro de las primeras 24 horas. Se instaló catéter de Swan-Ganz observándose un perfil compatible con SCPH (índice cardíaco de $2,18 \mathrm{~L} / \mathrm{min} / \mathrm{m}^{2}$, presión de oclusión en la arteria pulmonar $12 \mathrm{mmHg}$ y resistencia vascular sistémica indexada 1137 dinas.seg. $\mathrm{cm}^{5} \cdot \mathrm{m}^{2}$ ). Doce horas más tarde, se complementó monitorización con el sistema PiCCO el cual mostró un volumen sanguíneo

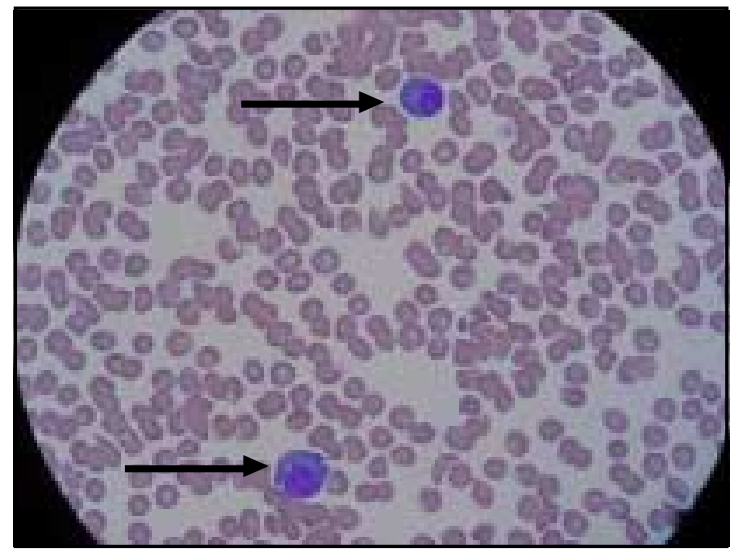

FIGURA 2. Inmunoblastos

intratorácico indexado (ITBVI intrathoracic blood volume index) normal $\left(1062 \mathrm{ml} / \mathrm{m}^{2}\right)$ y agua pulmonar extravascular indexada (EVLWI extravascular lung water index) alta $(14 \mathrm{ml} / \mathrm{kg})$. La IgM para virus Hanta resultó positiva, confirmado posteriormente por el Instituto de Salud Pública. En el segundo día de hospitalización el paciente se encontraba en falla renal aguda (creatinina 4,76 mg/dL, BUN $88 \mathrm{mg} / \mathrm{dLy}$ anuria) razón por la que se decidió iniciar hemofiltración de alto volumen (50 L de intercambio, $60 \mathrm{ml} /$ $\mathrm{kg} / \mathrm{h}$ ). En el tercer día se encontraba hemodinámicamente estable ( SOFA 10 puntos》) con menor apoyo de drogas vasoactivas (noradrenalina 0,14 $\mu \mathrm{g} / \mathrm{kg} /$ min) y bien adaptado a ventilación mecánica, mantenía una distensibilidad toraco-pulmonar de 56 $\mathrm{ml} / \mathrm{cmH}_{2} \mathrm{O}$, completando su tercera dosis de corticoides sistémicos. Se apreció paralelamente un descenso progresivo del EVLWI (de 14 a $6 \mathrm{ml} / \mathrm{kg}$ ) desde el día 1 al 5, con valores de ITBVI e índices cardíacos dentro de rangos normales, y reducción paulatina de agentes vasoactivos. La distensibilidad se mantenía en $56 \mathrm{ml} / \mathrm{cmH}_{2} \mathrm{O}$. Se inició alimentación por sonda nasoenteral. En el día 8 de hospitalización ( SOFA 5») se apreció un incremento discreto pero progresivo de la diuresis y función depurativa (creatinina 4,5 mg/dL y nitrógeno ureico $66 \mathrm{mg} / \mathrm{dL}$ ), suspendiéndose las drogas vasoactivas. Ocho días más tarde fue extubado y se suspendió la hemofiltración (creatinina 2,62 mg/dL y nitrógeno ureico 63 $\mathrm{mg} / \mathrm{dL}$ ), siendo trasladado de la unidad a los 18 días de su ingreso, y dado de alta del hospital a los 28 días. Un año y medio post-alta se encuentra integrado a su actividad habitual sin secuelas permanentes. 


\section{DisCUSIÓN}

La infección por Hantavinus tiene básicamente dos formas de presentación: síndrome hemorrágico renal descrito en Asia y Europa, y el SCPH descrito inicialmente en Estados Unidos. Su presencia en Chile se conoce desde 1995, en que se identificó al primer paciente. En nuestro medio el virus comesponde a la variedad Andes, cuyo reservorio es el ratón Oligoryzomys Longicaudatus. Su presencia se ha establecido desde el sector sur de la región metropolitana hasta las zonas más extrema del sur del país, donde se encuentra su mayor densidad poblacional.

La forma de presentación del SCPH en nuestro paciente, aunque inespecífica (fiebre, compromiso del estado general, mialgias marcadas, diarrea), fue bastante característica asociada a un importante antecedente epidemiológico.

Tanto en Chile como en el extranjero, la mortalidad descrita para este síndrome es de 40$70 \%{ }^{3,8}$ siendo fundamental en la recuperación de estos pacientes la terapia precoz de soporte de los diferentes sistemas orgánicos.

El presente caso es especialmente interesante, porque a diferencia de lo habitualmente comunicado, se trata de una persona mayor con importantes comorbilidades (HTA, DM 2), y constituye el primer caso de SCPH monitorizado con el sistema PiCCO. Al momento del ingreso el paciente presentó un APACHE II de 25 puntos y SOFA de
12 puntos, desarrollando rápidamente una falla multiorgánica, con una tasa de mortalidad predicha superior al $80 \% 9$.

Respecto del monitoreo hemodinámico invasivo se debe comentar que desde la introducción del catéter de arteria pulmonar por Swan y Ganz en 1970, la determinación del gasto cardíaco por termodilución y la presión de oclusión arterial pulmonar (POAP) han llegado a ser el sgold standard» en el manejo hemodinámico ${ }^{10}$. En el ambiente clínico, la POAP ha sido utilizada como indicador de la precarga cardíaca. No obstante, la relación entre ésta y el volumen telediastólico del ventrículo izquierdo no ha sido consistente. Factores como la presión intratorácica y la distensibilidad del ventrículo izquierdo pueden variar de una circunstancia a otra, como ocurre con los ajustes en la ventilación mecánica y el uso de catecolaminas.

En la actualidad, es posible estimar el gasto cardíaco y la precarga de manera más objetiva y precisa. Un importante aporte tecnológico ha sido el desarrollo de un sistema de medición de estos parámetros por medio de termodilución transcardiopulmonar. El PiCCO es un nuevo dispositivo que permite evaluar en la cabecera del paciente (latido a latido) el gasto cardíaco de manera continua mediante el análisis del contorno de la onda de pulso a nivel de la arteria femoral, braquial 0 axilar, y ofrece la posibilidad de conocer el ITBV y EVWL ${ }^{11-14}$. Se ha demostrado que la medición

Tabla 1. Parámetros hemodinámicos registrados por Swan-G anz y PiC C 0 y requerimiento de drogas vasoactivas

\begin{tabular}{|lccccccc|}
\hline Variables & \multicolumn{2}{c}{ DIA 1 } & DIA 2 & DIA 3 & DIA 4 & DIA 5 & VN \\
& Ingreso & 12 horas & & & & & \\
\hline IC $\left(\mathrm{L} / \mathrm{min} / \mathrm{m}^{2}\right)$ & 2,18 & 3,74 & 2,75 & 3,45 & 2,85 & 2,82 & $2,6-4,0$ \\
POAP $(\mathrm{mmHg})$ & 12 & 10 & 14 & 19 & 14 & 17 & $6-12$ \\
RVSI $\left(\mathrm{dinas}\left(\mathrm{seg} / \mathrm{cm}^{5} / \mathrm{m}^{2}\right)\right.$ & 1137 & 1690 & 1833 & 2226 & 1543 & 1787 & $1200-2500$ \\
ITBVI $\left(\mathrm{ml} / \mathrm{m}^{2}\right)$ & & 1062 & 892 & 837 & 840 & 839 & $850-1000$ \\
EVLWI $(\mathrm{ml} / \mathrm{kg})$ & & 14 & 11 & 10 & 7 & 6 & $4-7$ \\
PAM $(\mathrm{mmHg})$ & 78 & 90 & 77 & 82 & 68 & 80 & $70-105$ \\
NA $(\mu \mathrm{g} / \mathrm{kg} / \mathrm{min})$ & - & 0,29 & 0,20 & 0,14 & 0,09 & 0,05 & \\
DOPA $(\mu \mathrm{g} / \mathrm{kg} / \mathrm{min})$ & - & 5 & - & - & - & - & \\
DOBU $(\mu \mathrm{g} / \mathrm{kg} / \mathrm{min})$ & - & - & 4 & 2,5 & 2,5 & - & \\
SOFA & 12 & & 12 & 10 & 9 & 9 & \\
\hline
\end{tabular}

IC: índice cardíaco, POAP: presión de oclusión en la arteria pulmonar, RVSI: resistencia vascular sistémica indexada, ITBVI: volumen sanguíneo intratorácico indexado, EVLWI: agua pulmonar extravascular indexada, PAM: presión arterial media, NA: noradrenalina, DOPA: dopamina, DOBU: dobutamina, VN: valores normales 
Tabla 2. Evolución temporal de la medición del EVLW I y los parámetros de oxigenación

\begin{tabular}{|lcccccc|}
\hline Variables & DIA 1 & DIA 2 & DIA 3 & DIA 4 & DIA 5 & VN \\
\hline EVLWI $(\mathrm{ml} / \mathrm{kg})$ & 14 & 11 & 10 & 8 & 6 & $4-7$ \\
$\mathrm{PaFi}$ & 165 & 196 & 187 & 191 & 241 & $>300$ \\
$\mathrm{IO}\left(\mathrm{cmH} \mathrm{C}_{2} \mathrm{O} / \mathrm{mmHg}\right)$ & 9,5 & 8,1 & 7,5 & 7 & 6 & $<2$ \\
$\mathrm{DS}\left(\mathrm{ml} / \mathrm{cmH}_{2} \mathrm{O}\right)$ & 52 & 55 & 56 & 58 & 61 & $50-100$ \\
$\mathrm{BH}(\mathrm{ml})$ & +2663 & +2465 & +659 & +183 & +969 & \\
$\mathrm{PCR}(\mathrm{mg} / \mathrm{dL})$ & 35 & 27,3 & 4,7 & 2,6 & 2,3 & $<2$ \\
\hline
\end{tabular}

EVLWI: agua pulmonar extravascular indexada, PaFi: presión arterial de oxígeno/fracción inspirada de oxígeno, $\mathrm{IO}_{2}$ : índice de oxigenación, DS: distensibilidad estática toraco-pulmonar, BH: balance hídrico, PCR: proteína C reactiva, VN: valores normales

del ITBV es mejor indicador de la precarga cardíaca que la POAP y la presión venosa central (PVC). Además, esta variable se ve menos influenciada por las presiones intratorácicas y las variaciones de la distensibilidad ventricular izquierda, especialmente en los pacientes hemodinámicamente inestables ${ }^{15,16}$. En el presente caso, el paciente fue evaluado mediante los dos sistemas de monitoreo en un intento por definir con mayor precisión el perfil hemodinámico y orientar las medidas de reanimación. Pudimos constatar que las mediciones intermitentes del índice cardíaco por Swan-Ganz y el registro continuo del índice cardíaco con el sistema PiCCO eran bastante similares. Por el contrario, no existió un buen acuendo entre el ITBVI y la POAP (Tabla 1). En la evaluación del contenido de agua pulmonar, el sistema PiCCO reveló un patrón compatible con edema de permeabilidad, característico del SCPH con EVLWI elevado e ITBVI normal.
La reanimación con fluidos, si bien fue efectiva en optimizar la estabilidad hemodinámica, indujo durante las primeras 48 horas un balance hídrico positivo de aproximadamente $5 \mathrm{lt}$; sin embargo, a pesar de no conseguirse un balance hídrico francamente negativo en las siguientes $72 \mathrm{~h}$, el EVLWI y los parámetros de intercambio gaseoso $\left(\mathrm{PaO}_{2} / \mathrm{FiO}_{2}\right.$, Indice de Oxigenación) se fueron normalizando (Tabla 2), con una radiografía de tórax que mostraba menos infiltrados intersticiales y alveolares, a lo que se asoció una caída paulatina en la PCR (como índice de actividad inflamatoria) (Figura 3). Es posible que la hemofiltración de alto volumen haya contribuido al mejoramiento hemodinámico del paciente durante la fase más crítica de su evolución, permitiendo reducir la intensidad del soporte vasopresor y optimizar el manejo de fluidos. Este efecto ha sido reportado previamente en casos de shock séptico severo ${ }^{17,18}$ y estaría asociado a una mayor remoción de mediadores pro-inflamatorios.

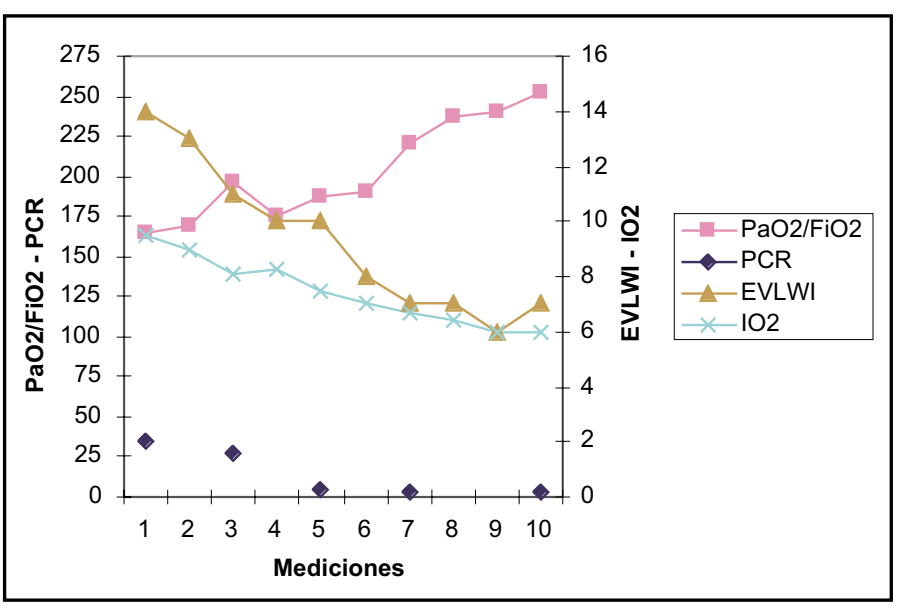

FIgURA 3. Comportamiento del EVLW y parámetros de oxigenación en el tiempo. Nótese la relación entre la evolución del EVLWI y la PCR.

$\mathrm{PaO}_{2} / \mathrm{FiO}_{2}$ : presión arterial de oxígeno/ fracción inspirada de oxígeno, PCR: proteína C reactiva, EVLWI: agua pulmonar extravascular indexada, $\mathrm{IO}_{2}$ : índice de oxigenación. 
El empleo de corticoides puede haber jugado un rol importante en la estabilización de membranas y modulación de la respuesta inflamatoria sistémica. Actualmente se encuentra en marcha un estudio chileno-norteamericano Fase II para tratar de establecer la utilidad de los esteroides en esta patología.

En resumen, el síndrome cardiopulmonar, representa el grado extremo de la infección por hantavirus, con una rápida progresión del deterioro

\section{REFERENCIAS}

1. Mertz GJ, Vial PA. Emergencia del síndrome cardiopulmonar por hantavirus en las Américas. Rev Chil Infect 2000; 17: 181-5.

2. Hawin GW, Simpson SQ, Crowell RE, James DS, Koster FT, Mertz GJ, LeVY H. Cardiopulmonary manifestations of hantavirus pulmonary syndrome. Crit Care Med 1996; 24: 252-8.

3. Duchin JS, Koster FT, Peters CJ, Simpson GL, Tempest B, ZAKI SR ET AL. Hantavirus pulmonary syndrome: A clinical description of 17 patients with a newly recognized disease. N Engl J Med 1994; 330: 94955.

4. Crowley M, Katz R, Kessier R, Simpson SQ, Levy H, HALIN GW ET AL. Successful treatment of adults with severe Hantavirus pulmonary syndrome with extracorporeal membrane oxygenation. Crit Care Med 1998; 26: 409-14.

5. Sotomayor V, Aguilera X. Epidemiología de la infección humana por hantavirus en Chile. Rev Chil Infect 2000; 17: 220-32.

6. Navarrete M, Saldías F, Mancila R, Zaror L, Ferres M. Evidencia clínico epidemiológica de la existencia de síndrome pulmonar por hantavirus en Valdivia-Chile desde 1993. Rev Chil Infect 2000; 17: 233-40.

7. Castillo C, Naranjo J, Ossa G, Levy H. Hantavirus pulmonary syndrome due to Andes virus in Temuco, Chile: clinical experience with 16 adults. Chest 2001; 120: 548-54.

8. Tapia M, Mansila C, Vera J. Síndrome pulmonar por hantavirus: Experiencia clínica en diagnóstico y tratamiento. Hospital Coyhaique-Chile. Rev Chil Infect 2000; 17: 258-69.

9. López F, Peres D, Bross A, Mélot C, Vincent JL Serial evaluation of the SOFA score to predict outcome in critically ill patients. JAMA 2001; 286: 1754-8.

10. CRUz K, FRANKLIN C. The pulmonary artery catheter: respiratorio y cardiovascular. En la actualidad no existe una terapia específica para esta patología. El monitoreo y análisis de la información obtenida con el sistema PiCCO podría modificar la conducta terapéutica, posibilitando un manejo más fino y proporcionado de la resucitación con fluidos, optimizando el empleo de drogas vasoactivas y guiando la terapia depletiva con diuréticos o procedimientos dialíticos, medidas que en definitiva podrían influir positivamente en la evolución de estos enfermos.

uses and controversies. Crit Care Clin 2001; 17: 271-91.

11. Preisman S, Pfeiffer U, Lueberman N, Perel A. New monitors of intravascular volume: a comparison of arterial pressure waveform analysis and the intrathoracic blood volume. Intensive Care Med 1997; 23: 651-7.

12. SakKa SG, Rühl CC, Pfeiffer UJ, Beale R, McLuckie A, Reinhart K, Meier-Helumann A. Assessment of cardiac preload and extravascular lung water by single transpulmonary thermodilution. Intensive Care Med 2000; 26: 180-7.

13. Hudson E, BEALE R. Lung water and blood volume measurements in the critically ill. Curr Opin Crit Care 2000; 6: 222-6.

14. Gödje O, HöKe K, Goetz A, Felbinger T, Reuter D, REICHART B ET AL. Reliability of a new algorithm for continuous cardiac output determination by pulsecontour analysis during hemodynamic instability. Crit Care Med 2002; 30: 52-8.

15. Lichtwarck-Aschoff M, Beale R, Pfeiffer UJ. Central venous pressure, pulmonar artery occlusion pressure, intrathoracic blood volume and right ventricular end-diastolic volume as indicators of cardiac preload. J Crit Care 1996; 11: 180-6.

16. SakKa SG, Bredie DL, Reinhart K, Meier-Heumann A. Comparison between intrathoracic blood volume and cardiac filling pressures in the early phase of hemodynamic instability of patients with sepsis or septic shock. J Crit Care 1999; 14: 7-83.

17. Honore PM, Jamez J, Wauthier M, Lee PA, Dugernier T, PIRENNe B ET al. Prospective evaluation of shortterm, high-volume isovolemic hemofiltration on the hemodynamic course and outcome in patients with intractable circulatory failure resulting from septic shock. Crit Care Med 2000; 28: 3581-87.

18. Cole L, Bellomo R, Journois D, Davenport P, Baldwin I, TIPPING P. High-volume haemofiltration in human septic shock. Intensive Care Med 2001; 27: 978-86. 